\title{
DNA Replication and Sister Chromatid Cohesion 1 (DSCC1) of the Replication Factor Complex CTF18- RFC is Critical for Colon Cancer Cell Growth
}

\author{
Jong-Tae Kim ${ }^{1 *}$, Hee Jun Cho ${ }^{1 *}$, Sang Yoon Park ${ }^{1}$, Byung Moo Oh',2, Yo Sep Hwang ${ }^{1,2}$, Kyoung Eun Baek ${ }^{1}$, \\ Young-Ha Lee ${ }^{3}$, Hee Cheol Kim ${ }^{\natural 凶}$ and Hee Gu Lee ${ }^{1,2 \bowtie}$ \\ 1. Immunotherapy Research Center, Korea Research Institute of Bioscience and Biotechnology (KRIBB), Daejeon, Republic of Korea. \\ 2. Department of Biomolecular Science, University of Science and Technology (UST), Daejeon, Republic of Korea. \\ 3. Department of Infection Biology, Chungnam National University School of Medicine, Daejeon, Republic of Korea \\ 4. Department of Surgery, Samsung Medical Center, Sungkyunkwan University School of Medicine, Seoul, Republic of Korea. \\ *These authors contributed equally to this work.
}

$\triangle$ Corresponding authors: Hee Cheol Kim, M.D., Ph.D., Department of Surgery, Samsung Medical Center, Sungkyunkwan University School of Medicine, Seoul, Republic of Korea. E-mail: hckim@skku.edu or Hee Gu Lee, Ph.D., Immunotherapy Research Center, Korea Research Institute of Bioscience and Biotechnology, Daejeon 34141, Republic of Korea. Tel: +82-42-860-4182; Fax: +82-42-860-4593; E-mail: hglee@kribb.re.kr

(C) The author(s). This is an open access article distributed under the terms of the Creative Commons Attribution License (https://creativecommons.org/licenses/by/4.0/). See http://ivyspring.com/terms for full terms and conditions.

Received: 2018.12.17; Accepted: 2019.08.26; Published: 2019.10.15

\begin{abstract}
DNA replication and sister chromatid cohesion 1 (DSCC1) combines with chromosome transmission-fidelity protein 18 (CTF18) to form a CTF18-DSCC1-CTF8 (CTF18-1-8) module, which in combination with CTF18-replication factor C (RFC) acts as a proliferating cell nuclear antigen (PCNA) loader during DNA replication-associated processes. It was found that $\mathrm{DSCCl}$ was overexpressed in tumor tissues from patients with colon cancer and that the survival probability of patients with colon cancer was lower when the expression of cytosolic DSCCl was higher in tumor regions $(\mathrm{P}=0.047)$. By using $\mathrm{DSCCl}-$ or CTF18-knockdown cell lines (HCT116-shDSCC1 or HCT116-shCTF18, respectively), it was confirmed that DSCC1-knockdown inhibits cell proliferation and invasion, but that CTF18-knockdown does not. Tumors in mice xenografted with shDSCCl cells were significantly smaller compared with those in mice in the mock group or those xenografted with shCTF18 cells. The shDSCCl cells were highly sensitive to $\mathrm{Y}$-irradiation and other DNA replication inhibitory treatments, resulting in low cell viability. The present results suggested that DSCCl is the most important component in the CTF18-1-8 module for CTF18-RFC and is highly relevant to the growth and metastasis of colon cancer cells, and, therefore, it may be a potential therapeutic target for colon cancer treatment.
\end{abstract}

Key words: DSCC1, CTF18, CTF18-1-8 module, colon cancer, metastasis

\section{Introduction}

Colon cancer is the third most common malignancy and the fourth leading cause of cancer-associated mortality worldwide $[1,2]$, with $>1$ million people diagnosed annually [3]. A recent study reported that $<600$ genes are linked to colon cancer-associated outcomes [4], indicating that the high expression of these genes relative to normal regulation is associated with poor outcomes. Accurate chromosome duplication and segregation is vital to maintaining genome integrity, but stressors associated with DNA replication-related processes can cause tumorigenesis and may represent major drivers of genomic instability $[5,6]$.

Replication factor C (RFC), which is essential for DNA replication, comprises 5 subunits (Rfc1-5). The four pentameric RFC complexes [Rfc1-RFC, Elg1-RFC, Rad24-RFC, and chromosome transmission-fidelity protein 18 (CTF18)-RFC] contain four common subunits (Rfc2-5) and a single, variable, large subunit that determines the roles of the complex [7]. The prototypical member (Rfc1-RFC) is essential for all replication and repair processes, whereas Rad24-RFC is involved in the DNA-damage checkpoint response [8-10], and Elg1-RFC is involved in proliferating cell 
nuclear antigen (PCNA) unloading [11] and genomic stability [12-14]. CTF18-RFC was originally identified in screens for chromosome mis-segregation [15-17] in budding yeast (Saccharomyces cerevisiae) and is important for the establishment of sister-chromatid cohesion [16, 18] and the activation of the replication-stress checkpoint $[15,19,20]$. During DNA-replication processes, CTF18-RFC assists DNA replication by loading the PCNA clamp onto the DNA [21-23], but the depletion of CTF18-RFC reduces the speed of replication-fork traversal [24] and results in defects in telomere metabolism [25] and DNA-repair processes [26, 27].

Unlike other RFCs, the CTF18-RFC complex includes the CTF18-DSCC1-CTF8 module (CTF18-1-8) $[21,22]$, wherein two non-RFC subunits [DNA replication and sister chromatid cohesion 1 (DSCC1) and CTF8] combine. The CTF18-1-8 module alone cannot affect the catalytic activity of the PCNA clamp loader [28, 29], but it does associate with the leading-strand DNA polymerase $\varepsilon$ (Pol $\varepsilon$ ) [30-32]. Grabarczyk et al [21] reported that Pol $\varepsilon$ binds to the C-terminal winged-helix domain of DSCC1, which is the predicted double-stranded DNA (dsDNA)binding site, suggesting that the heterotetrameric complex (CTF18-1-8-Pol $\varepsilon)$ competitively blocks binding of CTF18-1-8 to dsDNA, resulting in two alternative pathways for recruiting CTF18-RFC to replication sites. Few studies have investigated the association between CTF18-RFC and tumors. Nonsynonymous somatic mutations, nucleotide mutations that alter the amino acid sequence of a protein, have been identified in the CTF18 gene (G2035A, C2560T; 1.9\%) of endometrial tumors [33], and CTF8 levels are reportedly lower in renal and prostate tumors [34]. Yamaguchi et al [35] demonstrated that DSCC1 is frequently upregulated in colon cancer cells, and these elevated DSCC1 levels confer chemoresistance to colon cancer cells. They also reported the location of DSCC1 in the nucleus and cytosol, but did not analyze the function according to DSCC1 position. Recently, it has been published that DSCC1 is important for the proliferation and prognosis of hepatocellular carcinoma [36].

The present study examined DSCC1 overexpression in tissues from patients with colon cancer, revealing that the survival probability of patients with elevated cytosolic DSCC1 expression was lower compared with that of patients displaying localized DSCC1 expression in the nucleus. Establishment of DSCC1- or CTF18-knockdown cell lines demonstrated that DSCC1 knockdown inhibited cell proliferation and invasion, whereas CTF18knockdown had little effect. In a mouse xenograft model, transfer of DSCC1-knockdown cells resulted in smaller tumors compared with those in controls, whereas transfer of CTF18-knockdown cells resulted in the formation of granular tumors. The present findings suggested that DSCC1 may be an important component of the CTF18-1-8 module associated with colon cancer progression and a promising therapeutic target for colon cancer treatment.

\section{Materials and Methods}

\section{Patient samples}

Colorectal cancer (CRC) samples were obtained from patients who underwent routine surgery at the Department of Samsung Medical Center (Seoul, South Korea). All patients were advised of the procedures and provided written informed consent, as approved by the Institutional Review Board of the Samsung Medical Center. Inclusion and exclusion criteria for the CRC cohort were defined as follows: patients were included when patients were histologically confirmed as having CRC and had not received chemotherapy before surgery, and the date of death or survival data were available. Patients were excluded when histopathologic data were incomplete and date of patient death or survival data had not been recorded, and patients who received chemotherapy were also excluded. All cases were collected from specimens resected between 2005 and 2010. The clinical status of each patient was classified according to the pathological grade of the tumor, tumor size, lymph node involvement, and Dukes' staging system for colon cancer. For the immunohistochemical study, colon cancer tissues and normal mucosal tissues taken from a site distant from the tumorous region were fixed in a $10 \%$ neutralized buffered formalin solution for $24 \mathrm{~h}$.

\section{Cells and reagents}

Human CRC cell lines (HCT116, SW480, SW620, LoVo, COLO205, KM12C, KM12SM, HT29, DLD1 and LS174T) were cultured in Dulbecco's modified Eagle's medium (Gibco; Thermo Fisher Scientific, Inc., Waltham, MA, USA), supplemented with heat-inactivated 10\% fetal bovine serum (FBS; Gibco; Thermo Fisher Scientific) and antibiotics $(100 \mathrm{U} / \mathrm{ml}$ penicillin and $100 \mathrm{mg} / \mathrm{ml}$ streptomycin), and maintained at $37^{\circ} \mathrm{C}$ in an incubator containing a humidified atmosphere of $5 \% \mathrm{CO}_{2}$. To establish stabilized DSCC1- or CTF18-knockdown cell lines, short hairpin RNA (shRNA) for DSCC1 or CTF18 (Mission shRNA; Sigma-Aldrich; Merck KGaA, Darmstadt, Germany) was transfected into Lenti-X 293T cells (Clontech Laboratories, Mountain View, CA, USA) using the lentiviral packaging mix (Sigma-Aldrich; Merck KGaA); the virus enrichment 
media was added to HCT116 or SW480 cells, and puromycin $(4 \mu \mathrm{g} / \mathrm{ml})$-resistant cell lines were selected.

\section{Reverse transcription-quantitative polymerase chain reaction ( $R T-q P C R$ )}

Colon cancer cell lines and normal/tumorpaired tissues from patients with colon cancer were lysed using TRIzol ${ }^{\circledR}$ reagent (Ambion; Thermo Fisher Scientific, Inc.), and the total RNA was isolated according to the manufacturer's instructions. Each cDNA was synthesized by using ProSTAR First-Strand RT-PCR kit (Stratagene, San Diego, CA, USA) at $42^{\circ} \mathrm{C}$ for $1 \mathrm{~h}$. Primers for DSCC1 (sense, 5'-CGTGGTGATAAAGACGAGCA-3'; antisense, 5'CCGGAGTTTTACAACCAGGA-3') and GAPDH (sense, 5'-CAATGACCCCTTCATTGACC-3'; antisense, 5'-GACAAGCTTCCCGTTCTCAG-3') were used. Snail, Slug, and E-cadherin primers were used for the analysis of epithelial-mesenchymal transition (EMT). PCR was performed in a ProFlex PCR system (Applied Biosystems; Thermo Fisher Scientific, Inc.) and qPCR was performed in QuantStudio3 (Applied Biosystems; Thermo Fisher Scientific, Inc.).

\section{Immunohistochemistry}

Tissue specimens from therapeutic procedures were fixed in formalin buffer and embedded in paraffin wax. Tissue sections (4- $\mu \mathrm{m}$ thickness) were deparaffinized, and antigen retrieval was conducted in citrate buffer. The sections were treated with 3\% hydrogen peroxide in methanol to quench the endogenous tissue peroxidase activity, followed by incubation with $1 \%$ BSA to block nonspecific binding. The sections were incubated with mouse anti-DSCC1 antibody (1:500 dilution; produced from a mouse immunized with DSCC1 C-terminal protein; Fig. S1) for $60 \mathrm{~min}$ at room temperature in a wet chamber. Following washing, the tissue section was reacted with biotinylated anti-mouse secondary antibody, and counterstained with $10 \%$ Mayer's hematoxylin. An unrelated mouse IgG of the same isotype or antibody dilution solution served as a negative control. Areas of most intense and predominant staining pattern were scored. The cytosolic and nuclear staining of DSCC1 was determined separately for each specimen. The staining intensity (SI) was graded as follows: 0 , no staining; 1 2, weak staining; 3 5, moderate staining; 6 9, intense staining (Tables I, III, S1, S2, S5, and S6). In each case, the staining was scored as an average throughout the spot. Scoring of DSCC1 was performed by two independent pathologists, and the average score was obtained for cases of disagreement.

\section{Plasmids, transfection, western blotting and antibodies}

Full-length DSCC1 (GenBank acc. no. NM_024094) and CTF18 (NM_022092), and CTF8 (NM_001039690) cDNAs were obtained from the Korea Human Gene Bank (KRIBB, Daejeon, South Korea), and cloned into the peGFPN2/C2 (Clontech Laboratories, Inc., Mountainview, CA, USA) and pcDNA3.1MycHis vectors (Invitrogen; Thermo Fisher Scientific, Inc.). All plasmid constructs were verified by DNA sequencing, and protein expression was verified by western blotting. For transfection, cells were plated 1 day prior and cells were transfected with Lipofectamine ${ }^{\circledR} 3000$ reagent (Invitrogen; Thermo Fisher Scientific, Inc.) according to the manufacturer's instructions, and 2 days subsequently the cells were lysed with radioimmunoprecipitation assay buffer containing a protease inhibitor cocktail (Sigma-Aldrich; Merck KGaA) on ice for $30 \mathrm{~min}$. A total of $30 \mu \mathrm{g}$ of protein was separated by $10-14 \%$ SDS-PAGE and then transferred using a Transblot Turbo transfer system (Bio-Rad Laboratories, Inc., Hercules, CA, USA). The membranes were blocked with 5\% skim milk/PBS and incubated with the appropriate primary antibodies and HRP-conjugated secondary antibodies at room temperature. Protein bands were visualized using enhanced chemiluminescence detection reagents (EMD Millipore, Billerica, MA, USA) and the Ez-Capture MG system (Atto Corporation, Tokyo, Japan). Polyclonal DSCC1 antibody was produced from $\mathrm{BALB} / \mathrm{c}$ mice immunized with the purified recombinant DCC1 C-terminal protein (Fig. S1). Anti-E-cadherin, CTF18, GAPDH, PCNA and MSH2 (mutS homolog 2) antibodies from Santa Cruz Biotechnology, Inc. (Dallas, TX, USA), anti-poly (ADP) ribose polymerase (PARP), Cyclin-D1 and c-Myc antibodies from Cell Signaling Technology, Inc. (Danvers, MA, USA), anti-caspase- 3 and -7 antibodies from Calbiochem (Merck KGaA), and anti-tubulin and -His monoclonal antibodies from Sigma-Aldrich (Merck KGaA) were used.

\section{Cell proliferation assay}

To examine the cell proliferation, $1 \times 10^{4}$ cells were plated in a 96-well plate, and 2 days later WST1 (Roche Applied Science, Penzberg, Germany) was added. After $2 \mathrm{~h}$ the absorbance at $450 \mathrm{~nm}$ was read using a multi-mode microplate reader (FilterMax F3; Molecular Devices, LLC, Sunnyvale, CA, USA).

\section{Clonogenic cell survival assay and flow cytometry}

Cells $\left(1 \times 10^{4}\right)$ were plated in $60-\mathrm{mm}$ dishes, and 1 day later the cells were $\gamma$-irradiated $\left(5 \mathrm{~Gy},{ }^{60} \mathrm{Co}\right)$. After 
2 weeks, the cells were stained with crystal violet (CV) and colonies were counted. For apoptosis analysis, cells were harvested, stained with a FITC-AnnexinV/Propidium Iodide (PI) apoptosis detection kit (BD Biosciences, San Jose, CA, USA) for $30 \mathrm{~min}$, and analyzed using FACSverse (BD Biosciences) and the FlowJo program (FlowJo LLC, Ashland, OR, USA).

\section{Xenograft assays}

HCT116-shRNAmock, -shDSCC1, or -shCTF18 cells were collected and washed with PBS, and 7×106 cells were injected subcutaneously into mice (6-8-week old, male BALB/c athymic nude mice; Orient Bio., Seongnam, South Korea). Tumors from mice were photographed and the weights were measured. Mice were maintained in accordance with the Guidelines and under the approval of the Institutional Review committee for Animal Care and Use (KRIBB AEC 17113).

\section{Cell migration and invasion assay}

Transwell plates (24-well; Corning Inc., Corning, NY, USA) were used, according to the manufacturer's instructions. Briefly, cells $\left(3 \times 10^{5}\right.$ cells $\left./ \mathrm{ml}\right)$ were plated in the upper wells containing serum-free media and after 2 days the cells were stained with CV. Cells remaining in the upper wells were removed using a cotton swab. Cells that had migrated through the $8-\mu \mathrm{m}$ pores were photographed and dissolved in $10 \%$ acetic acid, followed by measuring the absorbance at $560 \mathrm{~nm}$. Matrigel-coated Transwells were used for the invasion assay.

\section{Statistics}

All results were confirmed in at least three independent experiments and representative results are presented. The differences between groups were analyzed using a Student's t-test between two groups or by one-way analysis of variance. Disease-free survival (DFS) analyses were performed using multivariate Cox proportional hazards models accounting for age, sex, tumor stage, with colorectal cancer-specific 5-year follow-up, after which samples were right censored. Differences in survival were expressed as hazard ratio with 95\% confidence intervals (CI) and median survival time. Survival curves were calculated with the Kaplan-Meier method and the differences were estimated using the log-rank test. $\mathrm{P}<0.05$ was considered to indicate a statistically significant difference.

\section{Results} DSCC1 is overexpressed in tumor regions
from colon cancer patients

CTF18-1-8 module of the CTF18-RFC is involved in DNA replication and chromosome cohesionassociated processes. When the mRNA expression of the CTF18-1-8 module in colon cancer tissues was analyzed by qPCR, DSCC1 mRNA increased more strongly compared with CTF18 or CTF8 mRNA (data not shown). As presented in Fig. 1A, DSCC1 expression levels were higher in tumor tissues compared with normal tissues. Quantitative comparison of the mRNA expression of DSCC1 by qPCR ( $n=50$; Fig. 1B) revealed that the expression of DSCC1 at the tumor site was increased by $\sim 2.9$ times. To verify this, DSCC1 antibodies were generated in BALB/c mice immunized with recombinant DSCC1 C-terminal protein (Fig. S1). DSCC1 protein was expressed strongly in all colon cancer cell lines examined in the present study (Fig. S2A). To determine whether DSCC1 overexpression in patients with colon cancer $(n=206)$ was clinicopathologically significant, immunohistochemical staining of tumor tissues was performed. The results indicated that DSCC1 was localized to the nucleus and cytosol in tumor regions (Fig. 1C, Tables I, S1, and S2), with DSCC1 staining being particularly strong in the cytosol (Table I; P<0.001, cancer vs. normal). Examination of the clinicopathological characteristics indicated that although there was no significant association between DSCC1 levels in the cytosol (or nucleus) and patient sex, age, location, cell type, Tumor, Node, and Metastasis (TNM) stage (Tables S4-6), elevated cytosolic DSCC1 levels were significantly associated with microsatellite instability (MSI) status ( $\mathrm{P}=0.030$; Table II). Additionally, disease-free survival curves according to cytosolic (Fig. 1D) or nuclear (Fig. 1E) DSCC1 levels in stages II and III colon cancer revealed that cases with high cytosolic DSCC1 levels displayed better oncological outcomes $(\mathrm{P}=0.007)$, although there was no difference between high and low/negative nuclear DSCC1 levels $(\mathrm{P}=0.084)$. Univariate analysis for disease free survival (Table III) showed that the expression of DSCC1 was significant when comparing well differentiated tumors $(\mathrm{P}=0.016)$, and Cox regression analysis for the effect of several risk factors on survival (Table S7) showed that tumor N-stage $(\mathrm{P}=0.037)$ and cytosol-DSCC1 $(\mathrm{P}=0.031)$ were important compared to other factors.

Table I. DSCCl staining intensities (SI) of normal or colon cancer tissues

\begin{tabular}{llll}
\hline & Cancer $(\mathrm{n}=206)$ & Normal $(\mathrm{n}=33)$ & P value \\
\hline DSCC1_Cytosol & $2.93 \pm 1.87$ & $1.61 \pm 1.39$ & $<0.001$ \\
DSCC1_Nucleus & $2.96 \pm 1.77$ & $3.38 \pm 15.01$ & 0.870 \\
\hline
\end{tabular}


A

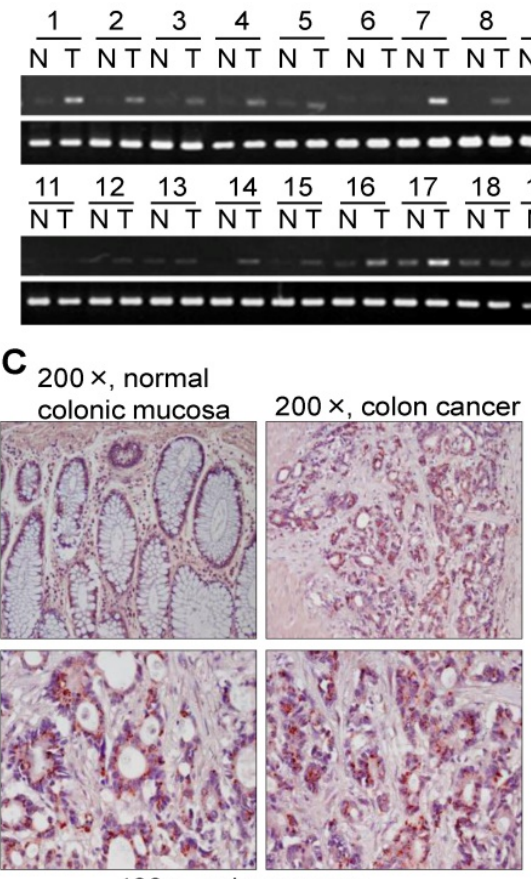

B

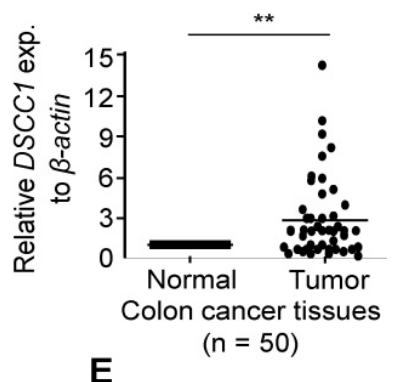

D

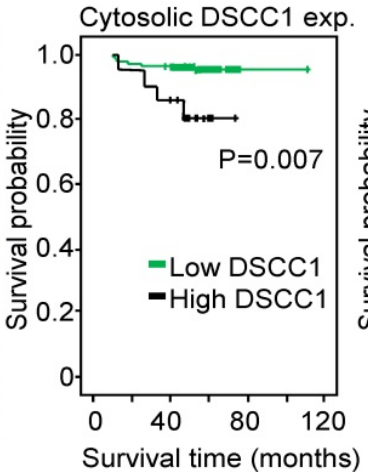

Nucleic DSCC1 exp

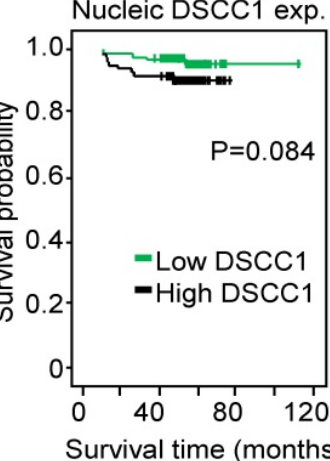

Figure 1. DSCC1 is overexpressed at the tumor site in patients with colon cancer. Whole RNA from normal (N)/tumor ( $T$ )-paired tissues from patients with colon cancer was extracted, their cDNAs were synthesized, and (A) RT-PCR or (B) qPCR was performed. $\beta$-actin was used as a reaction control. Data represent the mean \pm standard deviation of three independent experiments. ${ }^{* *} \mathrm{P}<0.01$. (C) Representative immunohistochemical staining of colon cancer tissues using the anti-DSCC1 antibody. As indicated in Materials and Methods, tissue sections were incubated with BSA, stained with anti-DSCCl antibody, and counterstained with hematoxylin. DSCCI was strongly stained in the cytosol at the tumor site. Disease-free survival curves according to (D) cytosolic or (E) nuclear DSCC1 levels in stages II and III colon cancer tissues. Cases exhibiting high cytosolic expression showed better oncological outcomes, but there was no difference between high and low/negative nuclear DSCC1 levels.

Table II. Clinicopathologic features according to cytosolic expression of DSCCI

\begin{tabular}{|c|c|c|c|c|}
\hline & Total & DSCC1 e & xpress & \\
\hline & $\mathrm{n}=206$ & Low/Negative & High & P value \\
\hline Sex & & & & 0.628 \\
\hline Male & 114 & 30 & 84 & \\
\hline Female & 92 & 21 & 71 & \\
\hline Age & & & & 0.633 \\
\hline $60>$ & 107 & 28 & 79 & \\
\hline $60 \leq$ & 99 & 23 & 76 & \\
\hline Location & & & & 1.000 \\
\hline Colon & 147 & 37 & 110 & \\
\hline Rectum & 59 & 14 & 45 & \\
\hline Cell type & & & & 0.107 \\
\hline High grade & 186 & 43 & 143 & \\
\hline Low grade & 20 & 8 & 12 & \\
\hline T stage & & & & 0.777 \\
\hline $\mathrm{T} 1 / \mathrm{T} 2$ & 18 & 5 & 13 & \\
\hline $\mathrm{T} 3 / \mathrm{T} 4$ & 188 & 46 & 142 & \\
\hline $\mathrm{N}$ stage & & & & 0.518 \\
\hline No & 107 & 24 & 83 & \\
\hline $\mathrm{N} 1,2$ & 99 & 27 & 72 & \\
\hline Stage & & & & 0.518 \\
\hline II & 107 & 24 & 83 & \\
\hline III & 99 & 27 & 72 & \\
\hline MSI status & & & & 0.030 \\
\hline MSI-H & 20 & 1 & 19 & \\
\hline MSI-L/MSS & 186 & 50 & 136 & \\
\hline
\end{tabular}

MSI, microsatellite instability; MSI-H, MSI high; MSI-L, MSI low; MSS, Microsatellite stable

\section{DSCC1 knockdown results in attenuated growth and invasiveness of colon cancer cells}

To investigate the function of DSCC1 overexpression in the tumor cells, DSCC1-knockdown cell lines were generated using shRNAs (Fig. S2B and C). Notably, HCT116-shDSCC1 cells exhibited a distinct mesenchymal morphology, different from that of shRNA-mock and shCTF18 cells (Fig. 2A), although SW480-shDSCC1 cells exhibited a weak mesenchymal morphology compared with HCT116-shDSCC1 cells (data not shown). Additionally, HCT116- and SW480-shDSCC1 cells grew more slowly compared with mock cells (Fig. 2B), and lower levels of cell cycle-associated cyclin D1 and cell adhesion-associated E-cadherin were observed (Fig. 2C). When cells were transiently transfected with DSCC1 plasmid and analyzed by luciferase reporter assay, promoter activities of activating protein 1 (AP1), E-cadherin, $\mathrm{T}$ cell factor (TCF) $/ \beta$-catenin were strongly increased (data not shown). In addition, we examined whether DSCC1 affects cell growth in cells including a normal colon cell line CCD841, gastric cancer cell lines AGS and SNU620, and embryonic kidney HEK293T cells. As in the case of CRC cell lines, DSCC1 showed strong expression in all of the examined cells (Fig. S3), and it was confirmed that the inhibition of DSCC1 by siRNA interferes with cell 
proliferation. Upon confirming that cell proliferation was slowed in the shDSCC1 cells, the present study examined whether cell invasion was also affected. Transcription factors (Snail, Slug) involved in the EMT process were downregulated (Fig. 2D), and the cell migration and invasion of shDSCC1 cells exhibited a $20-40 \%$ decrease (Fig. 2E and F). When DSCC1 was overexpressed exogenously in shDSCC1 cells, the cells exhibited similar activities of migration and invasion as the mock cells expressing DSCC1. These results suggested that DSCC1 is deeply involved in cell proliferation, migration and invasion.
Table III. Univariate analysis for disease free survival

\begin{tabular}{ll}
\hline Characteristics & P value \\
\hline DSCC1-cytosol (SI 0 vs. 1 8) & 0.007 \\
DSCC1-nucleus (SI 0 2 vs. 3 9) & 0.084 \\
& \\
Age (<65 vs. > or = 65) & 0.637 \\
Gender (M:F) & 0.457 \\
Tumor site (Colon:Rectum) & 0.669 \\
Differentiation & \\
WD vs. others & 0.016 \\
WD \& MD vs others & 0.795 \\
Lymph node metastasis (N:Y) & 0.264 \\
Stage (II:III) & 0.264 \\
MSI & 0.699
\end{tabular}

SI, staining intensity; WD, well differentiated; MD, moderately differentiated; MSI, microsatellite instability

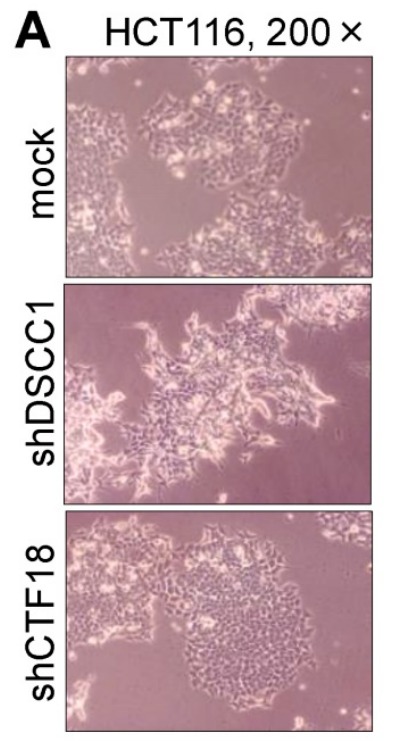

B

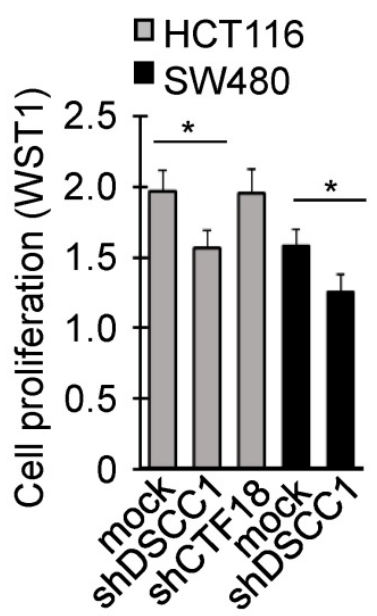

E
C

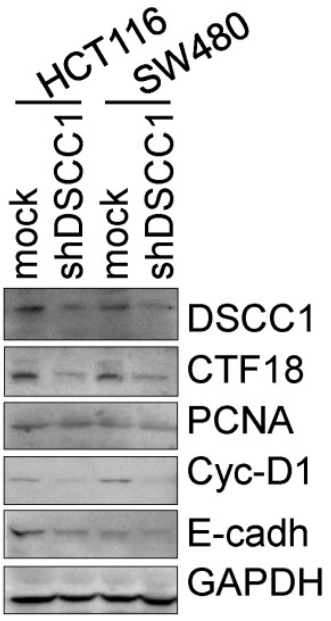

D

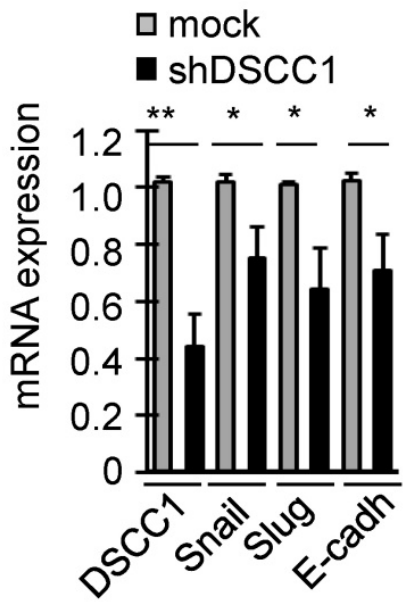

$\mathbf{F}$

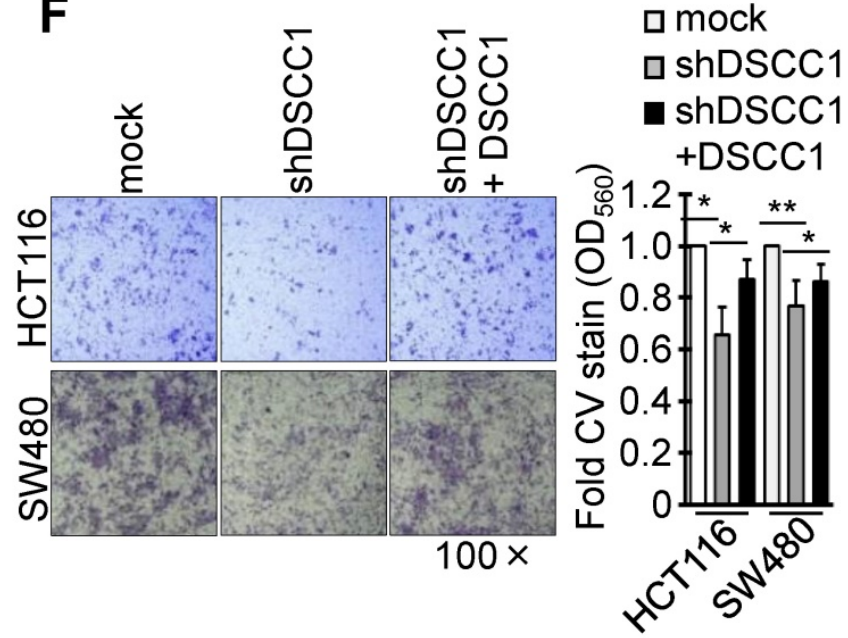

Figure 2. DSCCI knockdown slows the proliferation and invasion of colon cancer cells. (A) Image of HCT116-shDSCCI and -shCTF18 cells. Unlike mock and shCTF18 cells, shDSCC1 cells appeared to display a mesenchymal-like morphology. (B) HCT116-shDSCC1 cells exhibited slower cell proliferation compared with mock and shCTF18 cells. Cells were plated in 96-well plates. After 2 days WST1 added, and the plates were read at OD 450 . (C) Downregulation of cyclin-D1 and E-cadherin in shDSCC1 cells. Cells were harvested, and the lysates were subjected to SDS-PAGE followed by western blotting. (D) Downregulation of Snail and Slug genes in shDSCC1 cells. mRNAs were extracted from HCT116-shDSCC1 cells and qPCR was performed. GAPDH was used as a relative control. DSCC1 affected cell migration and invasion. Cells were added to the upper chambers of the Transwell chamber and (E) cell migration and (F) invasion abilities were analyzed. The migrated cells were stained with crystal violet (CV) to determine how many shDSCCl cells in the upper layer had passed through the (E) Transwell inserts or (F) Matrigel-coated membranes (F). CV-stained cells were dissolved in acetic acid and the absorbance was measured at $\mathrm{OD}_{560}$. To restore the expression of DSCC1, shDSCCl cells were transfected with PCDH-DSCCI plasmid. All data represent the mean \pm standard deviation of three independent experiments. ${ }^{*} P<0.05 ;{ }^{* *} P<0.01$. 

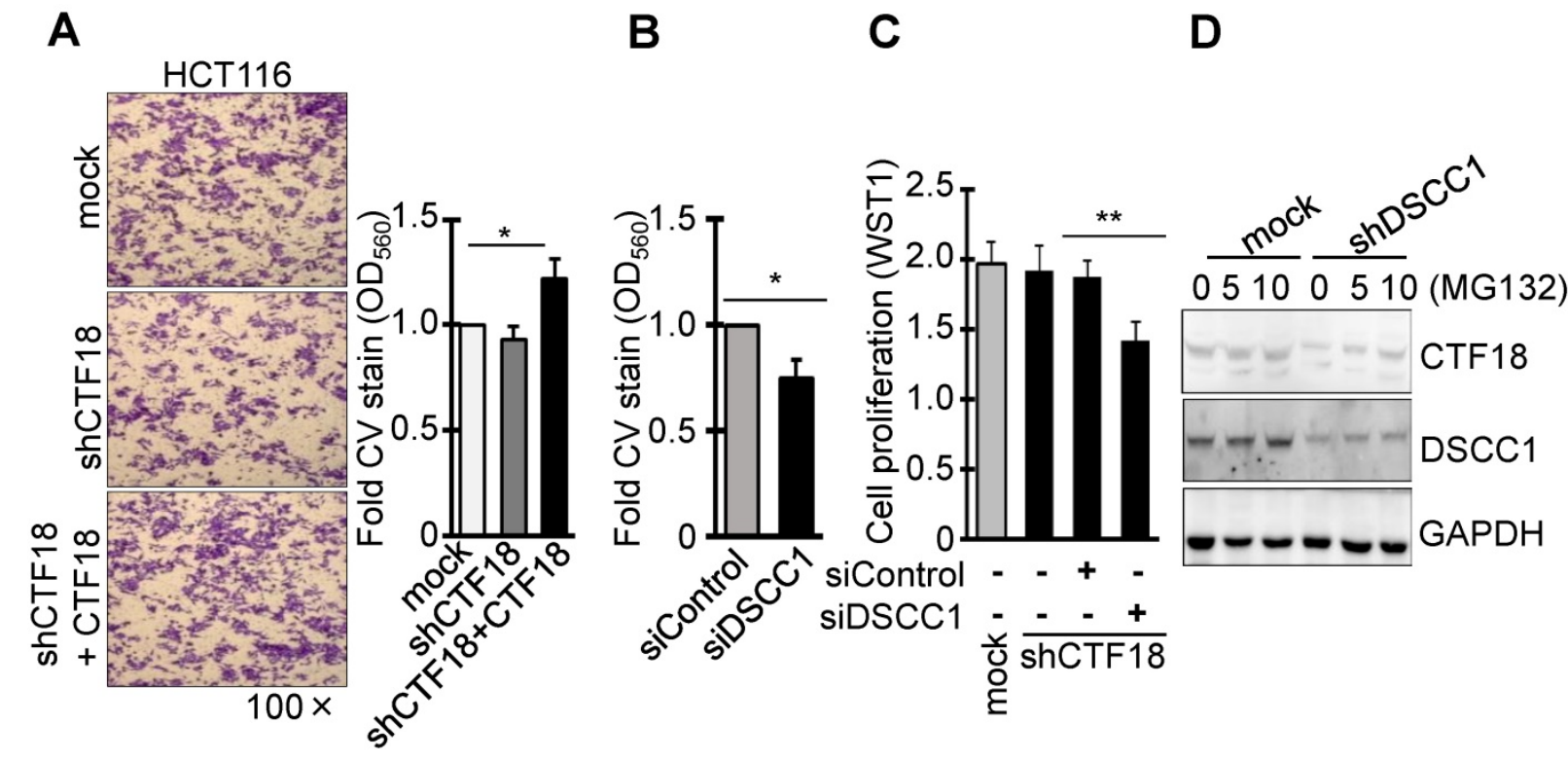

Figure 3. DSCC1 is more important for the CTF18-1-8 module than CTF18. (A) CTF18 knockdown did not affect cell invasion, but restoring CTF18 increased cell invasion. HCT116-shCTF18 cells were transfected with PCMV-CTF18 (or empty vector), loaded on the upper well, and 2 days later the cells that had penetrated the Matrigel-coated membrane were stained with CV; the absorbance was measured at OD 560 . (B) Decreased DSCC1, rather than CTF18 knockdown, effectively interfered with cell invasion. HCT116-shCTF18 cells were transfected with siDSCC1 (or control siRNA), and after 2 days the cells that had penetrated the Matrigel-coated membrane were stained with CV; the absorbance was measured at $\mathrm{OD}_{560}$. (C) DSCCl is more necessary for cell proliferation compared with CTF18. shCTF18 cells were transfected with siDSCCl (or control siRNA), and after 2 days WST1 was added and the plates were read at $\mathrm{OD}_{450}$. All data represent the mean \pm standard deviation of three independent experiments. ${ }^{*} \mathrm{P}<0.05 ;{ }^{* *} \mathrm{P}<0.01$. (D) $\mathrm{DSCC1}$ knockdown induced the proteolysis of CTF18. HCT116-shDSCC1 and -mock cells were treated with the proteasome inhibitor MG132 (5-10 $\mu \mathrm{g}, 48 \mathrm{~h})$, and cell lysates were subjected to SDS-PAGE followed by western blotting.

\section{DSCC1 is more important for colon cancer growth compared with CTF18 of CTF18-1-8 module}

It was identified that DSCC1 of the CTF18-1-8 module is important for CRC growth, and the present study also examined whether the binding of CTF18 to DSCC1 is important for colon cancer cell proliferation and invasion. Unlike DSCC1 knockdown, CTF18 knockdown did not inhibit cell proliferation (Fig. 2B) and invasion (Fig. 3A). However, shCTF18 cells transfected with CTF18 plasmid exhibited increased invasion, by $\sim 25 \%$ (Fig. 3A). Furthermore, DSCC1 siRNA (siDSCC1; Fig. S2E) transfection into shCTF18 cells confirmed that cell invasion (Fig. 3B) and proliferation (Fig. 3C) were reduced, as in shDSCC1 cells. Expression of CTF18 was reduced in shDSCC1 cells, but treatment with the proteasome inhibitor MG132 resulted in the gradual recovery of CTF18 expression (Fig. 3D). This suggested that DSCC1 knockdown may promote CTF18 degradation, eventually leading to the collapse of the CTF18-1-8 module. These results indicated that the role of DSCC1 of the CTF18-1-8 module is important for the cell proliferation and invasion of colorectal cancer.

\section{Inhibition of tumor formation in mice xenografted with DSCC1-knockdown cells}

To determine the tumor formation ability of shDSCC1 cells, HCT116-shDSCC1 cells were xenografted into athymic nude mice, and tumor sizes were examined. As presented in Fig. 4A, tumor formation in mice xenografted with shDSCC1 cells was significantly blocked compared with that in mice in the mock group. Notably, mice harboring HCT116-shCTF18 cells formed granule-shaped tumors distinct from those in the mock and shDSCC1 groups, presumably owing to a decrease in E-cadherin levels (Fig. 4A and C). Although transfer of either shDSCC1 or shCTF18 cells induced a decrease in E-cadherin levels in xenografted mice, tumor sizes between the two groups were very different (Fig. 4A and B). Also, CTF18 did not interfere with cell proliferation in vivo and in vitro, it led to the development of a different type of tumor compared with the normal control.

\section{DSCC1 is essential for the CTF18-1-8 module}

Since it is known that CTF18-RFC, which includes DSCC1, is involved in DNA replication and repair, the present study examined the degree of apoptosis in shDSCC1 cells treated with DNA replication-associated irradiation or chemotherapeutic drugs. To investigate the $\gamma$-irradiation-sensitivity of shDSCC1 cells, we performed clonogenic cell survival assays using treatment with 5 Gy irradiation (Fig. 5A). Compared with mock cells, $\sim 50 \%$ fewer shDSCC1 cell colonies were produced following irradiation, whereas $\sim 28 \%$ fewer shCTF18 cell colonies were produced. When 
shDSCC1 cells were treated with 10 Gy irradiation, shDSCC1 cells exhibited $24 \%$ more apoptosis compared with the mock group (Fig. 5B), and the expression of MSH2, which is involved in the DNA repair process, was not induced compared with the mock group (Fig. 5C). Additionally, when shDSCC1 cells were treated with chemotherapeutic drugs, such as hydroxyurea (HU), 5-fluorouracil (5-FU), doxorubicin, etoposide and cisplatin, shDSCC1 cells exhibited increased apoptosis (Fig. S4A), decreased cell proliferation (Fig. S4B), and increased S-phase arrest (Fig. S4C) compared with mock cells. Recovery of DSCC1 via transfection of a DSCC1 plasmid suppressed the activity of effector caspase-3 and -7 (Fig. S4D). Notably, CTF18 overexpression resulted in increased expression of DSCC1 in a dose-dependent manner in shDSCC1 cells (Fig. 5D), although exogenous DSCC1 did not induce CTF18 expression levels. Thus, when shDSCC1 cells were made to overexpress DSCC1 or CTF18, the number of cell colonies was increased compared to the control group (Fig. 5E), indicating that cell proliferation increases as the expression of DSCC1 is restored.

The cytosolic distribution of DSCC1 compared with the nucleus was clinicopathologically significant (Fig. 1D, Tables I-III and S5-S7). To investigate the effect of cytosolic DSCC1 on colon cancer cells, the DSCC1 mutant [the predicted nuclear-localization signal (NLS) of DSCC1 was mutated; DSCC1-NLSmut; Fig. S5A], expected to be located only in the cytosol, was prepared and compared with wild-type DSCC1. However, only $25 \%$ of DSCC 1 is located only in the cytosol, and the majority is present in the cytosol and nucleus (Fig. S5A and B). CTF18 and DSCC1 increased the irradiation-resistance of shDSCC1 cells, whereas overexpression of DSCC1-NLSmut resulted in weaker irradiation-resistance compared with DSCC1 or CTF18 overexpression (Fig. 5F). When comparing cell proliferation by overexpressing DSCC1 (or DSCC1-NLSmut), CTF18 and CTF8, as demonstrated with the radiation resistance, it was verified that the increase in DSCC1 expression also increased the cell proliferation (Fig. 5G). CTF8, constituting CTF18-1-8, did not affect cell proliferation. DSCC1-NLSmut, which has higher cytosolic distribution compared with DSCC1, exhibited a slight decrease in radiation resistance and cell proliferation. This result was likely due to the weaker CTF18-1-8 module for CTF18-RFC formation in the nucleus. To elucidate the role of DSCC1 in the cell cycle, shDSCC1 cells were synchronized to the G1 phase by serum deprivation for $24 \mathrm{~h}$ and subsequently treated with epidermal growth factor (EGF). As illustrated in Fig. $5 \mathrm{H}$, the induction of the expression of cyclin-D1 and c-Myc in shDSCC1 cells was less marked compared with that in mock cells, indicating that DSCC1 is essential for the role of CTF18-RFC in DNA replication during cell proliferation.

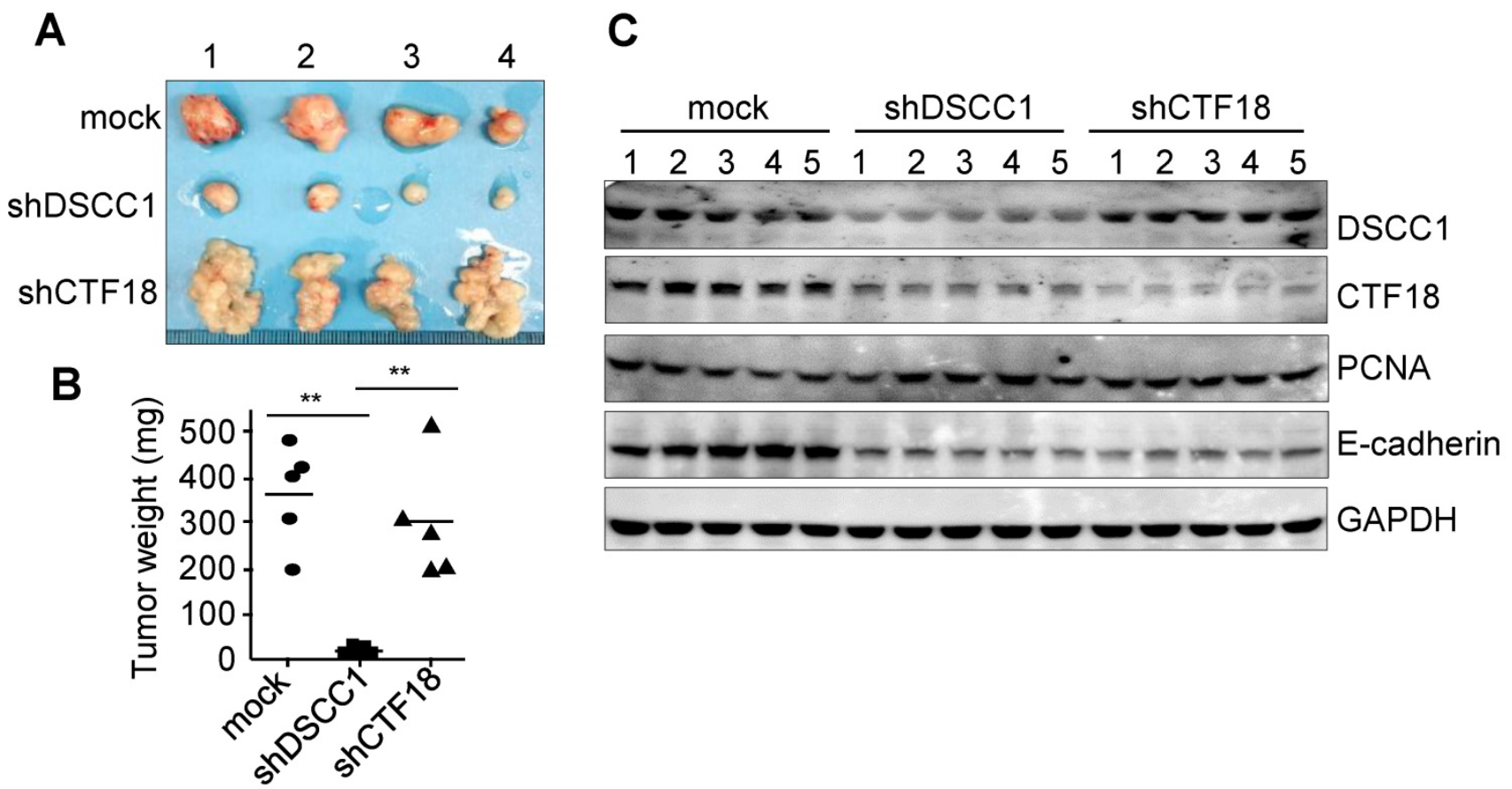

Figure 4. Inhibition of tumor formation in mice xenografted with DSCC1-knockdown cells. (A) HCT116-shDSCC1 or -shCTF18 cells were injected subcutaneously into athymic nude mice, and after 6 weeks tumors were isolated and photographed. shDSCCl cells significantly inhibited tumor formation, but shCTF18 cells formed granule-clump tumors. (B) The weight of tumor mass extracted from mice was measured. ** $P<0.01$.(C) Protein lysates prepared from tumor tissues were subjected to SDS-PAGE followed by western blotting. E-cadherin and CTF18 levels were attenuated in shDSCC1- and shCTF18-derived tumors. 
A

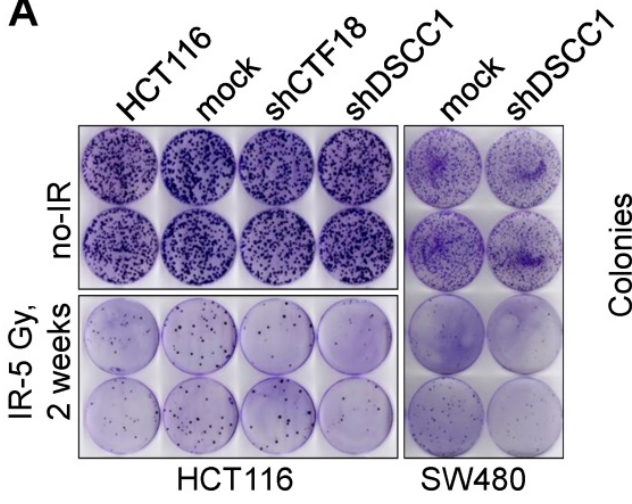

C

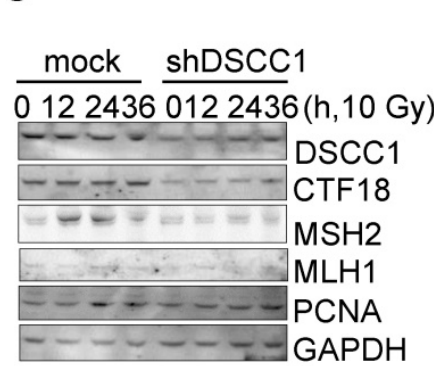

$\mathbf{F}$

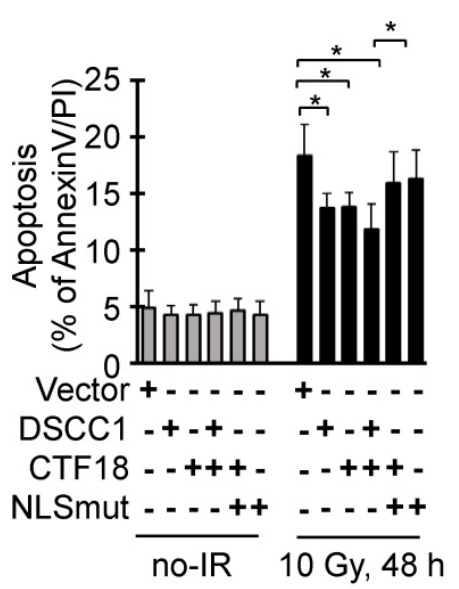

D

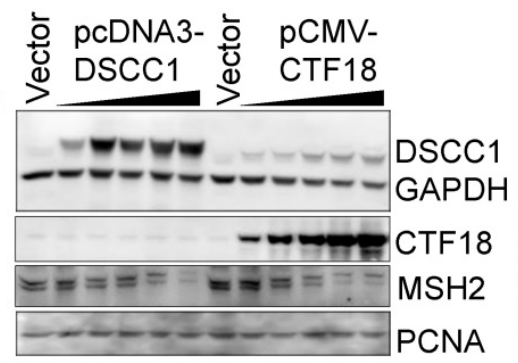

G

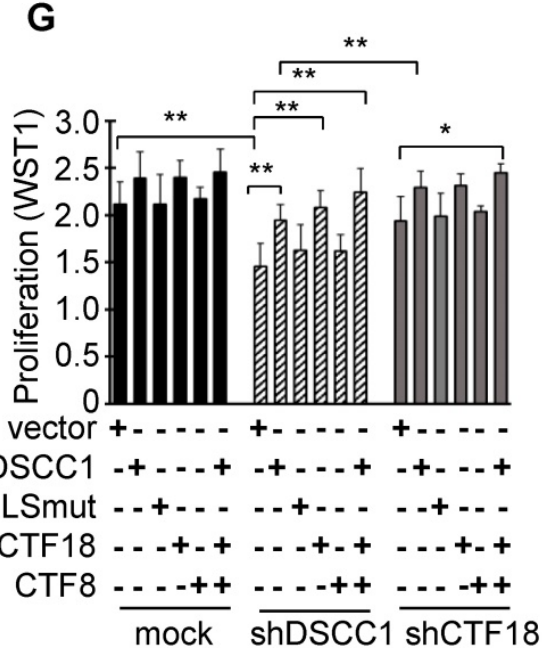

E
B
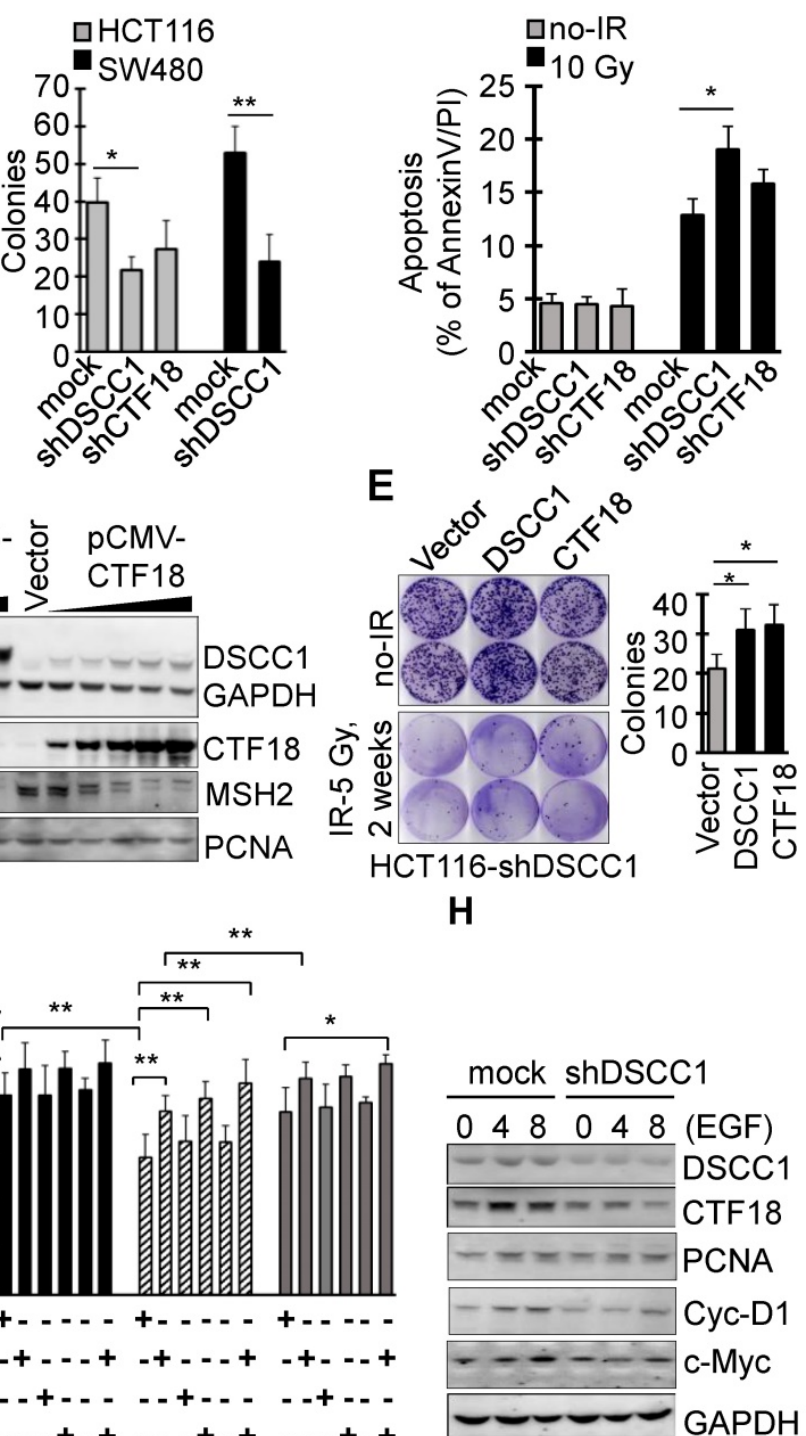

Figure 5. DSCCl is essential for the CTF18-1-8 module. (A) Clonogenic survival assays of shDSCC1 and shCTF18 cells. A total of $10^{3}$ cells in 60 mm dishes were $\mathrm{Y}$-irradiated with $5 \mathrm{~Gy}$, and after 2 weeks cells were stained with CV and colonies were counted. The survival rate associated with DSCC1 knockdown was lower. (B) DSCC1 knockdown promoted apoptosis. Cells were $\mathrm{Y}$-irradiated with $10 \mathrm{~Gy}$ for $48 \mathrm{~h}$, stained with Annexin-V and PI, and analyzed by flow cytometry. (C) DSCC1 knockdown inhibits MSH2 induction. Cells were $\mathrm{y}$-irradiated with $10 \mathrm{~Gy}$ for the indicated time, and cell lysates were subjected to SDS-PAGE followed by western blotting. Unlike the mock cells, DNA repair protein MSH2 was not induced in shDSCC1 cells. (D) CTF18 induced DSCC1 expression, but DSCC1 did not induce CTF18 expression. HCT116-shDSCC1 cells were transfected with pcDNA3-DSCC1 or PCMV-CTF18 plasmids, depending on the DNA concentration, for 2 days. Cell lysates were analyzed by western blotting. (E) Increased cell survival of DSCC1-rescued shDSCC1 cells was observed. shDSCC1 cells were transfected with DSCCI or CTF18 plasmids and $\mathrm{Y}$-irradiated with 5 Gy. After 2 weeks cells were stained with CV and colonies were count. (F) DSCC1 recovery is important for apoptosis resistance. $\mathrm{HCT} 116$-shDSCC1 cells were transfected with DSCC1 and/or CTF18 plasmids and $\mathrm{y}$-irradiated with $10 \mathrm{~Gy}$ for $48 \mathrm{~h}$, stained with Annexin-V and PI, and analyzed by flow cytometry. DSCC1 rescue by DSCC1 or CTF18 transfection led to apoptosis resistance, but DSCC1-NLSmut was less effective. (G) DSCC1 is the most important component of the CTF18-1-8 module. Cells were plated in a 96-well plate and transfected with DSCC1, DSCC1-NLSmut, CTF18 and/or CTF8 plasmids for 2 days. Cell proliferation was read at OD 450 . DSCC1 knockdown inhibited cell proliferation most significantly, but the recovery of DSCCl was effective at promoting cell proliferation. All data represent the mean \pm standard deviation of three independent experiments. ${ }^{*} \mathrm{P}<0.05$; ${ }^{* * P}<0.01$. (H) DSCC1 is required for the cell cycle protein Cyclin-D1. Cells were synchronized to the G1 phase by serum deprivation for 1 day, and subsequently treated with EGF $(20 \mathrm{ng} / \mathrm{ml})$ for $4-8 \mathrm{~h}$. Cell lysates were subjected to SDS-PAGE followed by western blotting.

\section{Discussion}

The present study verified the overexpression of DSCC1 in tissues from patients with colon cancer, along with clinicopathological findings indicating a lower survival probability in patients exhibiting elevated cytosolic DSCC1 levels. The immunohistochemical analysis demonstrated that DSCC1 was strongly expressed in the cytosol of tumor regions (Fig. 1C), whereas normal tissue displayed the majority of DSCC1 localized to the nucleus, with weak signals observed in the cytosol (Fig. 1C and S5). As presented in Table II, a significant correlation between MSI status in patients with colon cancer and 
elevated cytosolic DSCC1 levels was observed. However other clinicopathological features, such as sex, age, location, tumor grade and TNM stage, were not significant in patients exhibiting elevated cytosolic or nuclear DSCC1 levels (Tables). Moreover, the survival probability of patients exhibiting elevated cytosolic DSCC1 levels was lower compared with that of those with low cytosolic DSCC1 levels (Fig. 1D), whereas patients exhibiting elevated nuclear DSCC1 levels had no significant difference in survival probability (Fig. 1E). Yamaguchi et al [35] reported the nuclear and cytosolic localization of DSCC1 and the lack of a correlation between DSCC1 expression and clinicopathological features. To determine the role of cytosolic DSCC1 in tumorigenesis, the predicted NLS sequence of DSCC1 was mutated (DSCC1-NLSmut; Fig. S5). Although DSCC1-NLSmut was more distributed in the cytosol compared with the wild-type DSCC1, it did not significantly alter the cell proliferation or anti-apoptotic properties associated with DSCC1 overexpression (Fig. 5F and G). In addition, the predicted nuclear export signal (NES) of DSCC1, ${ }^{304}$ LDQLKGLAL ${ }^{312}$, was also mutated, but no significant differences were observed compared with DSCC1 and DSCC1-NLSmut (data not shown). The reason for the high cytosolic DSCC1 of patients with colon cancer could not be explained by DSCC1 mutants, but the tumorigenesis mediated by the cytosolic or nuclear localization of DSCC1 appears to be complicated.

A number of studies have performed experiments involving the knockdown or depletion of CTF18-RFC components. DSCC1-knockout clones are non-selectable by limiting dilution (while CTF18-RFCdeficient yeast grows robustly) and exhibit attenuated chromatid separation, indicating that this complex is essential in mammals [24]. Other studies demonstrated that a CTF18-deletion mutant was viable, but exhibited compromised chromosome cohesion and condensation [16, 17] and decreased Smc3 acetylation, leading to a defect in sister-chromatid cohesion [37], in addition to defects in cell proliferation, DNA-damage responses and genome stability [31]. The present study used DSCC1or CTF18-knockdown cell lines generated using shRNA lentiviral vectors (Fig. S2). Although the protein expression levels of DSCC1 or CTF18 were not completely suppressed relative to those in the controls, the present results indicated that DSCC1 knockdown significantly inhibited cell proliferation, clonogenic cell survival and invasion. However, CTF18-knockdown did not affect cell proliferation and invasion. Notably, when DSCC1- or CTF18knockdown cells were xenografted into athymic nude mice, shDSCC1 cells significantly inhibited tumor formation, whereas shCTF18 cells formed a mass of granules (Fig. 4).

Yamaguchi et al [35] reported that DSCC1 is important for the survival of cancer cells in response to $\gamma$-irradiation. In the present study, treatment of shDSCC1 cells with $\gamma$-irradiation or DNA-replicationassociated chemotherapeutic drugs, such as HU and 5-FU, increased cell death compared with mock cells (Fig. S4), with S-phase arrest specifically observed in shDSCC1 cells treated with HU. Additionally, it was observed that $\gamma$-irradiated shDSCC1 cells were more sensitive to apoptosis, and that clonogenic cell survival was lower compared with the controls (Fig. 5A). Notably, DNA-repair-associated MSH2 levels were not upregulated in shDSCC1 cells treated with $\gamma$-irradiation (Fig. 5B and C). As described earlier, MSI results from impaired DNA mismatch repair (MMR) as a consequence of germline mutations, such as those in MutL homolog 1 (MLH1), MSH2, MSH6, and PMS2 [38]. The present results indicated that MSH2 expression was induced by $\gamma$-irradiation-mediated MMR in control cells, but not in shDSCC1 cells, suggesting that DSCC1 is required for the MSH2-associated DNA-repair process.

DSCC1 knockdown caused CTF18 downregulation, and treatment with the proteasome inhibitor MG132 recovered CTF18 levels (Fig. 2D), indicating that attenuated DSCC1 levels promoted CTF18 degradation. The results of the present study and previous data [35] suggest that DSCC1 was unaffected by MG132 administration, and that DSCC1 stability is not likely to serve a major role in cancer cells. It was demonstrated that attenuated DSCC1 levels reduced CTF18 expression, decreased the speed of replication-fork traversal, and increased sister-chromatid collapse, suggesting that DSCC1 is important for DNA replication and recovery from genotoxic insult [24]. Examination of the role of CTF8 in DSCC1- or CTF18-knockdown cells showed that CTF8 did not enhance cell proliferation (Fig. 5G). A previous study reported that the DSCC1-CTF8 subcomplex is not required for PCNA-loading or -unloading reactions [28, 29], but that CTF8 is required for CTF18-RFC-associated activity at DNA replication forks [16, 25, 39] and activation of the S-phase checkpoint $[19,40]$. Although the present study did not identify the mechanism of PCNA loading affected by DSCC1 knockdown, the DSCC1 knockdown revealed that the cell cycle protein cyclin-D1 was induced later compared with the control (Fig. 5H), indicating that the low levels of DSCC1 and CTF18 affected the CTF18-1-8 module formation and development of functional CTF18-RFC. A recent study reported that disrupting either the clamp loading activity of CTF18-RFC or the integrity 
of the CTF18-1-8 module prevents the CTF18-RFC complex from fulfilling its cellular functions [31].

Although the direct function of DSCC1 in tumorigenesis has not been fully elucidated, the present results suggested that DSCC1 is the most important component of the CTF18-1-8 module for functional CTF18-RFC, and is important for the growth and metastasis of colon cancer, thereby making it a potential therapeutic target for colon cancer treatment.

\section{Supplementary Material}

Supplementary figures and tables. http://www.jcancer.org/v10p6142s1.pdf

\section{Acknowledgements}

The authors would like to thank the research assistants at the Laboratory Animal Resource Center, KRIBB.

\section{Funding}

This work was supported by the National Research Foundation of Korea (NRF) grant funded by the Korea government (No. 2017R1A2B2005629), the Korea Health Technology R\&D Project (Korea Health Industry Development Institute, the Ministry of Health \& Welfare; grant no. HI15C1678), and the Basic Science Research Program (the National Research Foundation of Korea, the Ministry of Science, ICT and Future Planning; grant no. NRF-2017R1A2B4012822).

\section{Authors' contributions}

JTK, HGL, and HCK conceived and planned the experiments. CRC tissue collection and analysis was performed by HCK and HGL. Clinicopathological analysis was performed by HCK. JTK, HJC, SYP, BMO, KEB, YSH, and HCK performed experiments. JTK, HJC, YHL, HCK and HGL contributed to the interpretation of the results. JTK and HGL took the lead in writing the manuscript. All authors provided critical feedback and helped shape the research, analysis and manuscript.

\section{Ethics approval and consent to participate}

The study was approved by the Institutional Review Board of the Samsung Medical Center, and was performed in accordance with the Declaration of Helsinki and current ethical guidelines. Prior informed consent was obtained from all participants.

\section{Competing Interests}

The authors have declared that no competing interest exists.

\section{References}

1. Favoriti P, Carbone G, Greco M, Pirozzi F, Pirozzi RE and Corcione F. Worldwide burden of colorectal cancer: a review. Updates Surg. 2016; 68: 7-11.

2. Arnold M, Sierra MS, Laversanne M, Soerjomataram I, Jemal A and Bray F. Global patterns and trends in colorectal cancer incidence and mortality. Gut 2017; 66: 683-691.

3. Cunningham D, Atkin W, Lenz HJ, Lynch HT, Minsky B, Nordlinger B and Starling N. Colorectal cancer. Lancet 2010; 375: 1030-1047.

4. Uhlen M, Zhang C, Lee S, Sjöstedt E, Fagerberg L, Bidkhori G, Benfeitas R, Arif M, Liu Z, Edfors F, et al. A pathology atlas of the human cancer transcriptome. Science 2017; 357: eaan2507.

5. Gaillard H, García-Muse T and Aguilera A. Replication stress and cancer. Nat Rev Cancer 2015; 15: 276-289.

6. Macheret $\mathrm{M}$ and Halazonetis TD. DNA replication stress as a hallmark of cancer. Annu Rev Pathol. 2015; 10: 425-448.

7. Majka J and Burgers PM. The PCNA-RFC families of DNA clamps and clamp loaders. Prog Nucleic Acid Res Mol Biol. 2004; 78: 227-260.

8. Green CM, Erdjument-Bromage H, Tempst P and Lowndes NF. A novel Rad24 checkpoint protein complex closely related to replication factor C. Curr Biol.2000; 10: 39-42.

9. Shimomura T, Ando S, Matsumoto K and Sugimoto K. Functional and physical interaction between Rad24 and Rfc5 in the yeast checkpoint pathways. Mol Cell Biol. 1998; 18: 5485-5491.

10. de la Torre-Ruiz MA, Green CM and Lowndes NF. RAD9 and RAD24 define two additive, interacting branches of the DNA damage checkpoint pathway in budding yeast normally required for Rad53 modification and activation. EMBO J. 1998; 17: 2687-2698.

11. Kubota T, Nishimura K, Kanemaki MT and Donaldson AD. The Elg1 replication factor C-like complex functions in PCNA unloading during DNA replication. Mol Cell 2013; 50: 273-280.

12. Smolikov S, Mazor Y and Krauskopf A. ELG1, a regulator of genome stability, has a role in telomere length regulation and in silencing. Proc Natl Acad Sci U S A. 2004; 101: 1656-1661.

13. Ben-Aroya S, Koren A, Liefshitz B, Steinlauf R and Kupiec M. ELG1, a yeast gene required for genome stability, forms a complex related to replication factor C. Proc Natl Acad Sci U S A. 2003; 100: 9906-9911.

14. Kanellis P, Agyei $R$ and Durocher D. Elg1 forms an alternative PCNA-interacting RFC complex required to maintain genome stability. Curr Biol. 2003; 13: 1583-1595.

15. Naiki T, Kondo T, Nakada D, Matsumoto K and Sugimoto K. Chl12 (Ctf18) forms a novel replication factor C-related complex and functions redundantly with Rad24 in the DNA replication checkpoint pathway. Mol Cell Biol. 2001; 21: 5838-5845.

16. Mayer ML, Gygi SP, Aebersold R and Hieter P. Identification of RFC(Ctf18p, Ctf8p, Dcc1p): an alternative RFC complex required for sister chromatid cohesion in S. cerevisiae. Mol Cell 2001; 7: 959-970.

17. Hanna JS, Kroll ES, Lundblad V and Spencer FA. Saccharomyces cerevisiae CTF18 and CTF4 are required for sister chromatid cohesion. Mol Cell Biol. 2001; 21: 3144-3158.

18. Wade BO, Liu HW, Samora CP, Uhlmann F and Singleton MR. Structural studies of RFCCtf18 reveal a novel chromatin recruitment role for Dcc1. EMBO Rep. 2017; 18: 558-568.

19. Crabbé L, Thomas A, Pantesco V, De Vos J, Pasero P and Lengronne A. Analysis of replication profiles reveals key role of RFC-Ctf18 in yeast replication stress response. Nat Struct Mol Biol. 2010; 17: 1391-1397.

20. Kubota T, Hiraga S, Yamada K, Lamond AI and Donaldson AD. Quantitative proteomic analysis of chromatin reveals that Ctf18 acts in the DNA replication checkpoint. Mol Cell Proteomics 2011; 10: M110.005561.

21. Grabarczyk DB, Silkenat S and Kisker C. Structural Basis for the Recruitment of Ctf18-RFC to the Replisome. Structure 2018; 26: 137-144.

22. Pellegrini L. Dual Roles of Ctf18-RFC: Loading the Clamp and Angling for the Polymerase. Structure 2018; 26: 1-2.

23. Lengronne A, McIntyre I, Katou Y, Kanoh Y, Hopfner KP, Shirahige K and Uhlmann F. Establishment of sister chromatid cohesion at the S. cerevisiae replication fork. Mol Cell 2006; 23: 787-799.

24. Terret ME, Sherwood R, Rahman S, Qin J and Jallepalli PV. Cohesin acetylation speeds the replication fork. Nature 2009; 462: 231-234.

25. Hiraga S, Robertson ED and Donaldson AD. The Ctf18 RFC-like complex positions yeast telomeres but does not specify their replication time. EMBO J. 2006; 25: 1505-1514.

26. Ogi T, Limsirichaikul S, Overmeer RM, Volker M, Takenaka K, Cloney R, Nakazawa Y, Niimi A, Miki Y and Jaspers NG. Three DNA polymerases, recruited by different mechanisms, carry out NER repair synthesis in human cells. Mol Cell 2010; 37: 714-727.

27. Ogiwara H, Ohuchi T, Ui A, Tada S, Enomoto T and Seki M. Ctf18 is required for homologous recombination-mediated double-strand break repair. Nucleic Acids Res. 2007; 35: 4989-5000.

28. Bermudez VP, Maniwa Y, Tappin I, Ozato K, Yokomori K and Hurwitz J. The alternative Ctf18-Dcc1-Ctf8-replication factor C complex required for sister chromatid cohesion loads proliferating cell nuclear antigen onto DNA. Proc Natl Acad Sci U S A. 2003; 100: 10237-10242.

29. Bylund GO and Burgers PM. Replication protein A-directed unloading of PCNA by the Ctf18 cohesion establishment complex. Mol Cell Biol. 2005; 25: $5445-5455$ 
30. Murakami T, Takano R, Takeo S, Taniguchi R, Ogawa K, Ohashi E and Tsurimoto T. Stable interaction between the human proliferating cell nuclear antigen loader complex Ctf18-replication factor C (RFC) and DNA polymerase \{epsilon\} is mediated by the cohesion-specific subunits, Ctf18, Dcc1, and Ctf8. J Biol Chem. 2010; 285: 34608-34615.

31. Okimoto H, Tanaka S, Araki H, Ohashi E and Tsurimoto T. Conserved interaction of Ctf18-RFC with DNA polymerase $\varepsilon$ is critical for maintenance of genome stability in Saccharomyces cerevisiae. Genes Cells 2016; 21: 482-491.

32. García-Rodríguez LJ, De Piccoli G, Marchesi V, Jones RC, Edmondson RD and Labib K: A conserved Pole binding module in Ctf18-RFC is required for S-phase checkpoint activation downstream of Mec1. Nucleic Acids Res. 2015; 43: 8830-8838.

33. Price JC, Pollock LM, Rudd ML, Fogoros SK, Mohamed H, Hanigan CL, Le Gallo $\mathrm{M}$, et al: Sequencing of candidate chromosome instability genes in endometrial cancers reveals somatic mutations in ESCO1, CHTF18, and MRE11A. PLoS One 2013; 8: e63313.

34. Sun M, Ma L, Xu L, Li J, Zhang W, Petrovics G, Makarem M, Sesterhenn I, Zhang $\mathrm{M}$ and Blanchette-Mackie EJ. A human novel gene DERPC on 16q22.1 inhibits prostate tumor cell growth and its expression is decreased in prostate and renal tumors. Mol Med. 2002; 8: 655-663.

35. Yamaguchi K, Yamaguchi R, Takahashi N, Ikenoue T, Fujii T, Shinozaki M, Tsurita G, Hata K, Niida A and Imoto S. Overexpression of cohesion establishment factor DSCC1 through E2F in colorectal cancer. PLoS One 2014; 9: e85750.

36. Xie XW, Wang XY, Liao WJ, Fei R, Cong X, Chen Q, Wei L, Chen HS, Wang Y. Effect of Upregulated DNA Replication and Sister Chromatid Cohesion 1 Expression on Proliferation and Prognosis in Hepatocellular Carcinoma. Chin Med J (Engl). 2018; 131: 2827-2835.

37. Borges V, Smith DJ, Whitehouse I and Uhlmann F. An Eco1-independent sister chromatid cohesion establishment pathway in S. cerevisiae. Chromosome 2013; 22: 121-134.

38. Buecher B, Cacheux W, Rouleau E, Dieumegard B, Mitry E and Lièvre A: Role of microsatellite instability in the management of colorectal cancers. Dig Liver Dis. 2013; 45: 441-449.

39. Gellon L, Razidlo DF, Gleeson O, Verra L, Schulz D, Lahue RS and Freudenreich $\mathrm{CH}$. New functions of Ctf18-RFC in preserving genome stability outside its role in sister chromatid cohesion. PLoS Genet. 2011; 7: e1001298.

40. Pan X, Ye P, Yuan DS, Wang X, Bader JS and Boeke JD. A DNA integrity network in the yeast Saccharomyces cerevisiae. Cell 2006; 124: 1069-1081. 